\title{
Agromemoria en escenarios de posconflicto: usos del suelo y café en Aguadas, Pensilvania y Samaná (Caldas, Colombia)
}

\author{
Paola Andrea Acosta-Nates ${ }^{\star}$, Camila Andrea Martínez-Calderón**, \\ Andrés Felipe Vásquez-Valencia ${ }^{* * *}$
}

Estudiante del Doctorado en Estudios Territoriales, Facultad de Ciencias Jurídicas y Sociales, Universidad de Caldas, Manizales, Colombia. Correo electrónico: paonates@gmail.com

** Abogada. Joven investigador de Colciencias, Universidad de Caldas, Manizales, Colombia.

Correo electrónico: camilamartinezcal@hotmail.com

*** Estudiante de pregrado en Ingeniería Agronómica, Universidad de Caldas, Manizales, Colombia.

Correo electrónico:

af.vasquez@hotmail.com

Recibido: 30 de septiembre del 2015 Aceptado: 9 de noviembre del 2015

Cómo citar este artículo: Acosta-Nates, P.A., Martínez-Calderón, C. A. y Vásquez-Valencia,

A. F. (2016). Agromemoria en escenarios de postconflicto: usos del suelo y café en Aguadas, Pensilvania y Samaná (Caldas, Colombia), Cooperativismo y Desarrollo, 24(108), 95-105. doi: http://dx.doi. org/10.16925/co.v24i108.1263

\section{Resumen}

Propósito: parte de establecer las implicaciones de la transición de cultivos de tradición (café) como estrategia de los pobladores para permanecer en sus territorios defendiendo lo que hemos denominado la agromemoria, que implica un trabajo real en términos del uso del suelo y el ecosistema. Metodología: la metodología descriptiva utilizada busca especificar las características de los actores locales apoyándose en la entrevista como instrumento de interrelación para conocer la interpretación y variación en los usos del suelo en lo que la gente local reconoce como el antes, el durante y el después del conflicto armado. Resultados: de lo anterior se infiere que, a pesar de que los pobladores locales establecen diversas estrategias para no ser desplazados de sus territorios, las transformaciones territoriales evidentes, principalmente en el cambio de los usos del suelo, tienen serias implicaciones sobre los ecosistemas y, por lo tanto, en los medios económicos para decidir quedarse a largo plazo. Conclusiones: las transformaciones territoriales generadas por el conflicto armado y por factores de tipo medioambiental impactan de manera directa tanto en los referentes socioculturales como en los sistemas productivos tradicionales de los actores locales.

Palabras clave: agromemoria, conflicto territorial, estrategia, usos de suelo. 


\title{
Agro-Memory in Post-Conflict Scenarios: Land Use and Coffee in Aguadas, Pensilvania and Samaná (Caldas, Colombia)
}

\begin{abstract}
Purpose: It starts by establishing the implications of the transition of traditional crops (coffee) as a strategy for inhabitants to stay in their territories defending what we have called agro-memory, which implies real work in terms of land use and ecosystem. Methodology: The descriptive methodology used seeks to specify the characteristics of local stakeholders relying on the interview as an interrelation instrument to know the interpretation and variation of land use in what local people recognize as the before, during and after armed conflict. Results: Based on the foregoing, it is inferred that, although local people establish various strategies in order not to be displaced from their territories, obvious territorial transformations, mainly changes in land use, have serious implications for ecosystems and, therefore, for the economic means to decide to stay in the long term. Conclusions: Territorial transformations caused by armed conflict and environmental factors directly impact both socio-cultural referents and traditional production systems of local actors.
\end{abstract}

Keywords: agro-memory, territorial conflict, strategy, land use.

\section{Agromemória em cenários de pós-conflito: usos do solo e café em Aguadas, Pensilvania e Samaná (Caldas, Colômbia)}

\section{Resumo}

Propósito: parte de estabelecer as implicações da transição de lavouras de tradição (café) como estratégia dos povoadores para permanecer em seus territórios defendendo o que denominamos a agromemória, que origina um trabalho real em termos do uso do solo e do ecossistema. Metodologia: a metodologia descritiva utilizada busca especificar as características dos atores locais apoiando-se na entrevista como instrumento de inter-relação para conhecer a interpretação e a variação nos usos do solo e no que a população local reconhece como o antes, o durante e o depois do conflito armado. Resultados: disso, infere-se que, apesar de a população local estabelecer diversas estratégias para não ser deslocada de seus territórios, as transformações territoriais evidentes, principalmente na mudança dos usos do solo, têm sérias implicações nos ecossistemas e, portanto, nos meios econômicos para que seus habitantes se estabeleçam por longo prazo. Conclusões: as transformações territoriais geradas pelo conflito armado e por fatores ambientais impactam de maneira direta tanto nos referentes socioculturais quanto nos sistemas produtivos tradicionais dos atores locais.

Palavras-chave: agromemória, conflito territorial, estratégia, usos do solo. 


\section{Introducción ${ }^{1}$}

El café se ha convertido en indudable referente de la dinámica sociocultural y productiva del departamento de Caldas. Sin embargo, el impacto de los conflictos territoriales en los que se encuentra el accionar armado y diversos factores ambientales contribuyeron a que los pobladores locales fueran desplazados temporal o definitivamente. En ambos casos, las estrategias de resistencia estuvieron relacionadas con el tránsito de cultivos tradicionales como el café, hacia otros sistemas productivos que les permitieran mayor movilidad, pero que en todo caso respaldaran los procesos de territorialización, entendidos como prácticas y discursos para delimitar, acotar, circunscribir los territorios y están acompañados, tal como se ha puesto en evidencia en el proyecto marco, de decisiones como frentear, resistir y re-apropiar socioculturalmente los espacios fisiográficos (Nates-Cruz et al.,).

En ese sentido, la paulatina transición de una práctica tradicional que como el café representa la base de la economía de las poblaciones y una serie de prácticas socioculturales que han pasado de generación en generación, permite advertir que, en principio, el arraigo y la construcción de la praxis territorial no están ancladas a la cultura cafetera propiamente, sino al apego mismo por la tierra. Lo anterior nos permite defender el concepto de agromemoria como estrategia de continuación del trabajo en el sector agropecuario, con rutas de sostenibilidad alternativas en el marco de sus dinámicas económicas y sobre todo de posibilidad de réplica de los tejidos culturales y sociales puestos en los territorios y que han sido afectados por los conflictos territoriales.

El actual panorama político del país, que establece rutas de postconflicto entendidas institucionalmente como dinámicas que permiten superar implicaciones ocasionadas principalmente en el marco del conflicto armado, son más bien entendidas

\footnotetext{
1 Este artículo forma parte de los productos del proyecto "Proceso de Territorialización de la memoria en escenarios de postconflicto. Caracterización, implicación y lineamientos de políticas en el orden local, regional y nacional", en el que el autor participó en calidad de estudiante de pregrado. Proyecto Coordinado por Beatriz Nates-Cruz. Grupo de Investigación TerritorialidadesICsH, Universidad de Caldas. En calidad de coautoras de este artículo participaron Camila Andrea Martínez Calderón, joven investigador de Colciencias y Paola Andrea Acosta Nates, co-investigadora del proyecto.
}

desde el proyecto marco como tramas cotidianas en busca de estrategias para constituir escenarios de superación propia (y apropiada) al conflicto armado - o al conflicto territorial- que, en últimas, termina como postura política o fortaleciendo el nivel político comunal, pero que no necesariamente parten de allí (Nates-Cruz et al., 2015).

De esta manera, la dinámica en la que los actores locales ${ }^{2}$ transitan de un cultivo a otro o crean formas asociativas vinculadas a la agromemoria, aunque en algunos casos puede tener implicaciones sobre los adecuados usos del suelo, es en todo caso la estrategia que realmente puede generar un panorama viable para el posconflicto en términos de resistencia.

\section{Metodología}

El proyecto de investigación al que se encuentra adscrito este artículo, tomó como Unidad Socioespacial de Análisis (USEA) la figura de los municipios de Aguadas, Pensilvania y Samaná; municipios del departamento de Caldas. Esta selección se basa, de un lado, en la territorialización de los actores armados: guerrilla de las FARC — Frente 47 (Pensilvania y Samaná) - y paramilitares del Frente Cacique Pipintá (Aguadas) y, de otro lado, de ciertos conflictos de tipo ambiental, político y hasta fitosanitario que implicaron transición de cultivos tradicionales como el café a otras especies productivas entre 1997 y 2013.

El estudio se aborda utilizando el método descriptivo con el que según Bernal (2006). Se muestran, narran, reseñan o identifican hechos, situaciones, rasgos, características de un objeto de estudios, o se diseñan productos, modelos prototipos, guías, etcétera. La investigación descriptiva se guía por las preguntas de investigación que se formula el investigador, se soporta en técnicas como la encuesta, entrevista, observación y revisión documental (p. 113).

Está implementándola con líderes locales, asociaciones, representantes que han tenido esa pujanza por el campo, siendo personas que vivenciaron lo ocurrido se utilizó como instrumento la entrevista, que es una forma específica de interacción social que tiene por objeto recolectar datos para una indagación. El investigador, según Behar (2008), “formula

\footnotetext{
2 Se emplea el término actor local, que se refiere a las autodefiniciones de ellos mismos como campesinos, agricultores, términos que tienen una amplia connotación.
} 
preguntas a las personas capaces de aportarle datos de interés, estableciendo un diálogo peculiar, asimétrico, donde una de las partes busca recoger informaciones y la otra es la fuente de esas informaciones" (p. 55).

Para el caso de estudio, se formulan preguntas a los actores locales como: ¿usted nació aquí? ¿Qué especies agropecuarias ha tenido? ¿Por qué? ¿Existen o han existido procesos de asociatividad? ¿Cuáles? ¿Ha pensado en salir de aquí o por qué se queda? ¿Cómo maneja el uso del suelo?, lo que conlleva preguntarse sobre los procesos de permanencia y resistencia que denominamos aquí "agromemoria", en el marco de las estrategias para continuar con lo que tradicionalmente han cultivado o seleccionar otra opción de sustento, contextualizando lo que fue antes, durante y después de los conflictos territoriales que han afectado sus bases económicas y sociales.

La ventaja esencial de esta herramienta de investigación y en particular de la entrevista, reside en que son los mismos actores sociales quienes proporcionan los datos relativos a sus conductas, opiniones, deseos, actitudes y expectativas, cosa que por su misma naturaleza es casi imposible de observar desde fuera. Nadie mejor que la misma persona involucrada para hablarnos acerca de todo aquello que piensa y siente, de lo que ha experimentado o proyecta hacer (Kuhn, 2001, citado en Behar, 2008. p. 55).

Teniendo en cuenta lo anterior, el estudio se apoyó en abordajes metodológicos y enfoques teóricos provenientes de diferentes disciplinas sociales privilegiando lo cualitativo y esto tuvo como objetivo la recuperación por medio de la palabra, de la memoria, de las experiencias de los actores locales en torno a la agricultura, el conflicto territorial y la construcción de escenarios de postconflicto, que en buena medida posibilitaron su comprensión, y en consecuencia, brindó la posibilidad de triangular información. La triangulación es un vehículo que posibilita la "validación cruzada cuando dos o más métodos distintos resultan congruentes y proporcionan datos comparables" (Jick, 1979, citado en Garrido, 2004. p. 13); de este modo, se pudieron obtener datos (aunque no de una manera cuantitativa), dinámica que, en buena medida, permitió construir una red conceptual que se fue articulando con las narraciones, para poder así aportar una mirada de síntesis y reflexión en la comprensión del problema.

\section{Analisis y discusión}

La gran variedad climática y de suelos han hecho de Colombia un escenario con grandes potenciales agropecuarios, óptimos para el desarrollo y la reproducción de innumerables especies animales y vegetales, que, de acuerdo con las características del territorio, está dividido en seis regiones principalmente: Caribe, Insular, Pacífico, Orinoquía, Amazonía y Andina. Esta última, compuesta por los departamentos de Caldas, Risaralda y Quindío, constituye a su vez tierras de fertilidad media, con mucha profundidad y formadas bajo la influencia de cenizas volcánicas; potencial último que se advierte principalmente en Caldas por estar ubicado entre las cordilleras oriental, central y parte de la occidental. Sus características de heterogeneidad edafoclimáticas corroboran que, entre sus posibilidades de explotación agropecuaria, existe una gran diversificación de especies y sus usos de suelo han estado centrados en la agricultura, ganadería, turismo y minería.

A pesar de la multiplicidad de factores bióticos y abióticos que determinan los potenciales de los territorios, en el caso de Caldas, gran parte del desarrollo económico está particularmente ligado al cultivo de café, gracias a las características de los suelos que son derivados de cenizas volcánicas, además de la temperatura del departamento que varía de acuerdo con la altitud y el relieve, y se ve alterada por los vientos alisios del noreste y del sureste. Sobre el flanco oriental de la cordillera central se localizan los sectores más lluviosos, determinan las condiciones óptimas para el cultivo del café, que se constituye en el renglón básico de la economía departamental. Por tener acceso al río Magdalena, el departamento de Caldas puede explotar dicha vía fluvial como medio de comunicación particularmente adecuada para este renglón económico.

Todo lo anterior permitió convertir estos territorios en espacios óptimos y atractivos para el posicionamiento del café como la principal economía de la región, pese a la topografía y la dificultad para realizar labores en esta, anclado sin duda al proceso de colonización antioqueña y convirtiéndolo en condición misma de la dinámica social y cultural constructora de tejedura territorial. En ese sentido, la agromemoria comprende los acontecimientos ambientales, económicos, políticos y sociales sobre una especie que ha sido explotada culturalmente en un territorio y para la cual se crea un marco de posibilidades de tránsito a nuevas especies, como ocurre en este caso con el 
cultivo de café. La agromemoria pone en contexto la situación de desarrollo del cultivo en relación con la resistencia o fragilidad al cambio, y está vinculada con el arraigo cultural por parte de los actores locales.

A finales de la década del ochenta, se hizo especialmente notable el desplazamiento del cultivo de café por otros cultivos no solo en el departamento de Caldas sino a nivel nacional. Esta situación tuvo su origen en el rompimiento del pacto de cuotas que permitía establecer un equilibrio de precios entre los países productores del grano, además de la aparición de la broca (Hypothenemus hampei) "en 1988, en el sur de Nariño como una de las plagas que más afecta el café, adicionalmente, la reducción en la calidad del producto" (Bustillos y Cárdenas, 1991, citado en Bustillos, 2006. p. 1) y el ataque epidemiológico de la roya (Hemileia vastatrix) implicaron una serie de factores que juntos, generaron una reducción entre los años de 1987 y 1995 de hasta el 23\% de la producción acumulada de cuatro cosechas, llegando a niveles de $28 \%$ y $30 \%$ de pérdidas (Rivillas, Serna, Cristancho y Gaitán, 2011).

Los anteriores condicionamientos propios de políticas de tierras desestructuradas y de factores fitosanitarios incontrolados, permitían prever un mal panorama para este cultivo, circunstancia que fue aprovechada por parte de los grupos armados para expandirse y más tarde impulsar el desarrollo de cultivos de coca en Caldas (Caicedo, 19972007, p. 4), en especial, en lo referente a los frentes 9 y 47 de las Farc. Esta situación marcó determinantes cambios frente a la sustitución de los cultivos propios o de tradición en los municipios de estudio (Pensilvania, Samaná y Aguadas) y generó consecuencias, de un lado en las estrategias sociales y culturales de apropiación territorial y de otro frente a los usos adecuados del suelo. Ambas circunstancias resultan determinantes para identificar las estrategias de resistencia en medio de los conflictos territoriales que se desencadenan por múltiples factores. El primero de ellos corresponde al escenario de confrontación armada proveniente, especialmente, del departamento de Antioquia, que se asentó en Caldas a partir de los años 90, e involucró tanto a guerrilleros como a paramilitares de acuerdo con las implicaciones geoestratégicas de los territorios, y el segundo factor se relaciona con condiciones fitosanitarias, ambientales y de política de tierras, principalmente, que consiguen tener influencia directa con el fenómeno de cambios de uso del suelo y de afectación de la forma de vida de las comunidades.

\section{El conflicto armado en contexto: Pensilvania, Aguadas y Samaná Caldas}

Como la mayoría de los pueblos caldenses, Pensilvania hace parte de todo el contexto de la colonización antioqueña, cuya economía gira en torno al café y a la reforestación (que ocupa el segundo renglón de importancia), además del cultivo de caña panelera, plátano y ganadería extensiva. A través de su ubicación geográfica, es posible identificar el anclaje de los diversos grupos armados que incursionaron en el municipio: Pensilvania limita al norte y noroeste con los municipios de Sonsón y Nariño; al oriente con Samaná de donde proviene el Frente 47 de las FARC; al occidente, Aguadas, Salamina y Marulanda de donde proviene el bloque paramilitar Cacique Pipintá por la influencia de los límites con Antioquia y por el sur, limita con Marquetalia y Manzanares, sector influenciado por el Frente 47 de las FARC.

La presencia de dichos grupos al margen de la ley - guerrilla - en el municipio de Pensilvania se presenta alrededor de los años noventa, en historia común con los municipios circundantes y explicados a través de la ruptura del pacto mundial del café como estrategia para los actores armados para expandir e impulsar los cultivos de coca. Los cuatro corregimientos que componen el municipio (Arboleda, Bolivia, Pueblo Nuevo y San Daniel) han sido determinantes en la definición del conflicto armado, puesto que en ellos la presencia del Frente 47 de las FarC, unido al paramilitarismo moldeó dinámicas de desplazamiento y cultivo de hoja de coca, principalmente.

De otro lado, desde 1997, se denunciaron las inversiones por parte de los narcotraficantes, en la compra indiscriminada de tierras, donde Aguadas, Supía, Viterbo y La Dorada representan los municipios con mayor incidencia de este fenómeno. A través de un informe elaborado por la misión de observación electoral (Mayor, s. f.), se indica que:

Alrededor de las autodefensas, sobresale el tema de las relaciones entre mafia y política y se destacan las relaciones entre estructuras criminales rurales y urbanas, puesto que en Aguadas, por ejemplo, existen expresiones o vasos comunicantes con la oficina de cobro de La Terraza, con sede en años pasados en Envigado y con mucha influencia en Medellín. Lo anterior se expresa en la existencia de la banda o estructura criminal llamada "Los Cocholos", muy articulada al narcotráfico, que ha incidido en la política local y que alterna alianzas y disputas con 
el frente Cacique Pipintá por el control político del municipio (p. 6).

Bajo el mando del que localmente se concibe como el temido jefe paramilitar "Ernesto Báez", el bloque Pipintá se financiaba a través de dineros del narcotráfico y la cooptación histórica de las estancias políticas del municipio. Este fenómeno de cooptación política advierte dinámicas de anclaje, posicionamiento y accionar militar sustancialmente diferentes a las de otros municipios, toda vez que tales estructuras cuentan con el apoyo de todo un aparataje administrativo con el que ejercitan las acciones de su pleno interés.

Por su parte, el municipio de Samaná incursionó en el escenario de la confrontación política armada a partir de los años noventa con el accionar de la guerrilla a través de los Frentes 9 y 47 de las FARC, que se asentó primordialmente en los corregimientos de San Diego y Florencia, lo que trajo consigo la tentación de cultivos ilícitos. Ya para el 2000 incursiona el paramilitarismo por la parte centro y sur del municipio con los miembros de las Autodefensas Campesinas del Magdalena Medio y su comandante Ramón Isaza, buscando zonas medianamente altas para incursionar en el narcotráfico.

Las actividades económicas más afectadas con la irrupción del conflicto armado fueron las agropecuarias, principalmente porque los grupos al margen de la ley amenazaban a los campesinos para cambiar sus cultivos tradicionales como el café y el pan, por cultivos ilícitos. Los sectores industrial y comercial sufrieron de igual modo, pues los grupos armados intervinieron el transporte de los productos y los pobladores debían pagar vacunas.

En cada uno de los municipios aludidos, teniendo en cuenta la "diversidad de los pisos térmicos, Caldas ofrece una gran actividad agropecuaria, aunque limitada en algunas áreas por el relieve quebrado a escarpado y por las condiciones climáticas adversas" (Ministerio de Agricultura y Desarrollo Rural-madr, 2006. p. 24), donde las especies tales como café, caña panelera, plátano y frutales, destacados en la figura 1, constituyen la principal base económica de Caldas.



Figura 1. Economías propias o tradicionales, 1995-2003. Este mapa se publica en este artículo con la autorización de la directora del Proyecto tempo, marco del presente texto y el autor no tiene sobre él autoría alguna. Tomado de Procesos de territorialización de la memoria en escenarios de postconflicto. Caracterización, implicación y lineamientos de políticas en el orden local, regional y nacional, por Nates-Cruz et al., 2015. 
Pese a la importancia que revela la permanencia de un cultivo como factor que permite entender lo tradicional y lo estructural dentro de las comunidades, parte de la explicación de la transición de un cultivo a otro está ligada al impacto y las modalidades empleadas por los actores armados para establecer el control. En ese caso, la percepción del riesgo constante a ser desplazado o a perder sus tierras y activos productivos produce incentivos para el cambio en los usos del suelo. El uso del suelo puede experimentar diferentes modificaciones. Por un lado, es posible que aumente el porcentaje del suelo para la producción de cultivos transitorios, a costa de una reducción en los permanentes, incluso si estos últimos son más rentables que los primeros. Ello debido a que los cultivos transitorios dan cosechas más seguidas que los permanentes $y$, en muchos casos, requieren una menor inversión. Los cultivos transitorios permiten entonces a los hogares recuperar su inversión en un periodo más corto de tiempo y contar con efectivo permanentemente (Arias e Ibañez, 2012).
El cambio a otros cultivos puede ser una de las estrategias que posibilita a los pobladores continuar en sus territorios como se observa en la figura 2 ; en algunos de los casos es posible que haya resistencia a cambiar sus cultivos tradicionales, pese a ello la transición misma representa la posibilidad de resistencia y de adaptación de los pobladores a la dinámica de los actores armados. En el caso del corregimiento de Arma, municipio de Aguadas, por ejemplo, el cultivo de flores y la transición a frutales como aguacates y cítricos, da lugar a establecer cierto tipo de relación con los grupos armados (principalmente paramilitares) con la necesidad de los pobladores de modificar sus usos sobre la tierra que les permitiesen mayor movilidad dado el caso de confrontación, hostigamiento o amenaza; efecto mismo que puede evidenciarse en parte del municipio de Pensilvania.

El impacto de la transición de los sistemas productivos tradicionales, en particular del café, devela en principio un bosquejo sobre los cambios de

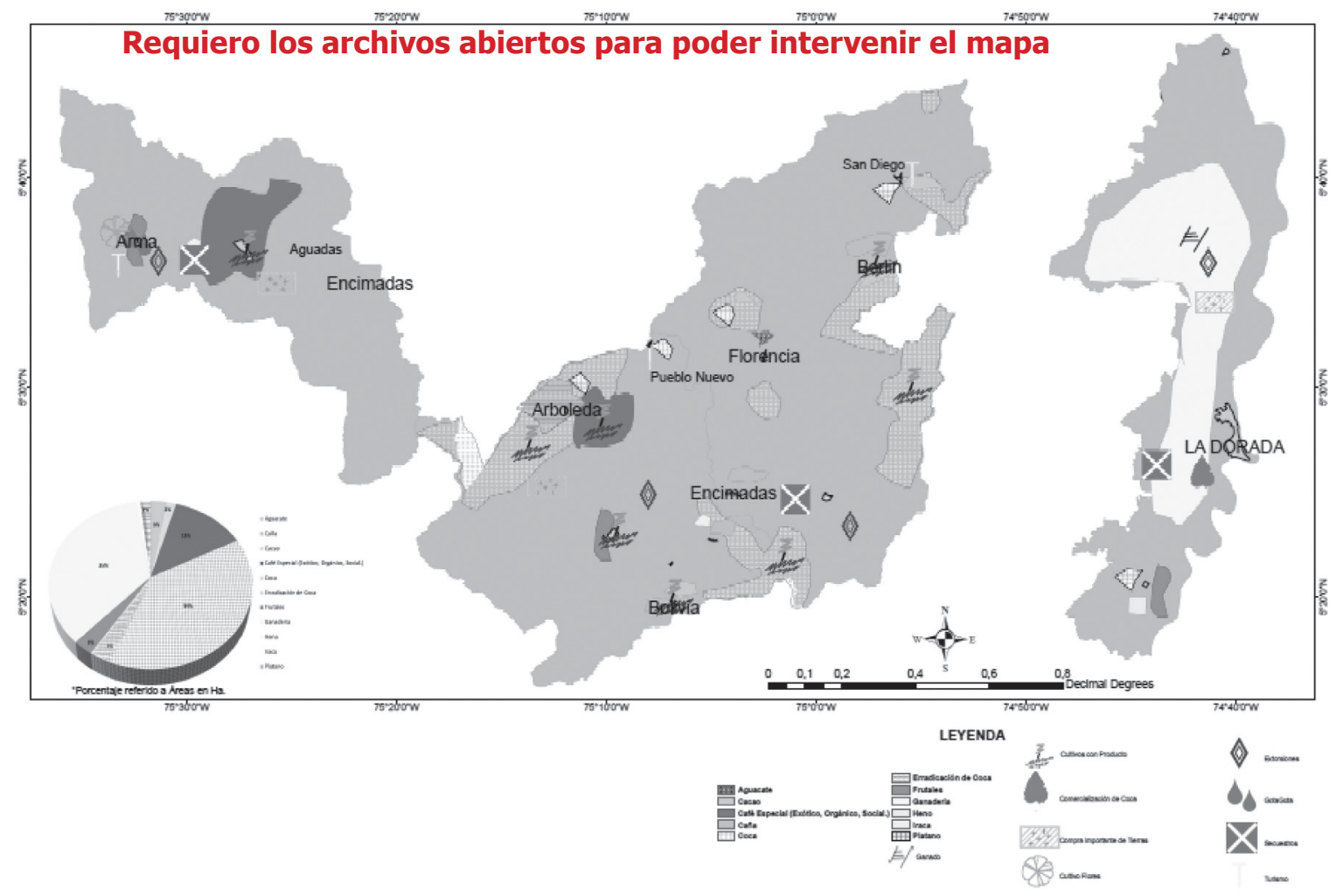

Figura 2. Economías apropiadas, 1995-2003. Este mapa se publica en este artículo con la autorización de la directora del Proyecto tempo, marco del presente texto y el autor no tiene sobre él autoría alguna. Tomado de Procesos de territorialización de la memoria en escenarios de postconflicto. Caracterización, implicación y lineamientos de políticas en el orden local, regional y nacional, por Nates-Cruz et al., 2015. 
decisiones de los pobladores ligadas estrechamente al conflicto armado circundante. En las bases de datos reportadas por el comité de cafeteros del 2013, entre los años de 1997 al 2003, se puede evidenciar un descenso en el área sembrada para el cultivo de café en relación con los sucesos de desplazamiento en el departamento de Caldas como se observa en la tabla 1; lo que, en principio, denota la relación inversamente proporcional de ambas variables así: a mayor desplazamiento forzado, menor número de hectáreas sembradas en café y a menor desplazamiento las hectáreas de café aumentan (tabla 1).

Tabla 1

Relación entre número de desplazados y área en café en el departamento de Caldas

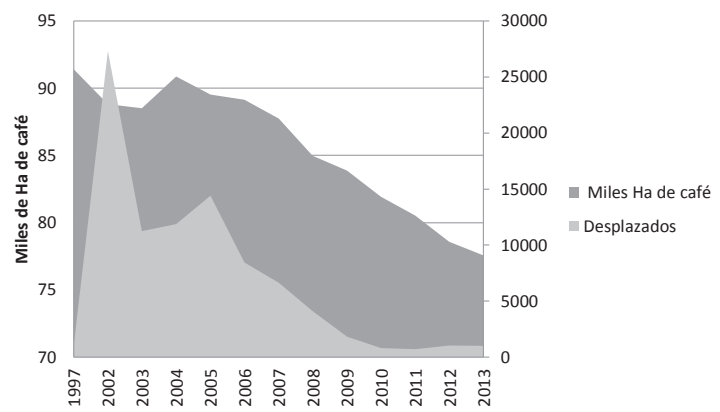

Nota. Adaptada de Áreas cultivadas y precio interno base del café en Colombia, por la Federación Nacional de Cafeteros Colombia, 2015, y Desplazamiento en Caldas, por Unidad de Víctimas, 2015.

Sin embargo, a partir del 2005, la relación de hectáreas de café sembradas en contraste con los niveles de desplazamiento se hacen directamente proporcionales; en ese caso a pesar de la disminución de la población desplazada, el cultivo de café también presenta disminución del área sembrada. Este fenómeno puede darse de un lado porque existe un cúmulo de población que, en todo caso, no retorna a sus territorios dado el escenario de confrontación armada y de otro, porque aunque habiendo retornado a sus territorios, los pobladores optan por establecer otro tipo de cultivos como alternativa que les permita mayor posibilidad de maniobra dado el caso de una nueva confrontación armada y porque hay un interés de los pobladores sobre la necesidad de cambio, toda vez que existe un impacto concomitante de factores por fuera del conflicto armado que determinan dicha decisión.
En el primer caso, la tabla 2 puede advertir una relación inversa en la que al reducirse la cantidad de personas desplazadas anualmente, el área sembrada en cultivos como la caña y el aguacate va en aumento de acuerdo con las condiciones edafoclimáticas y de manejo donde se esté dando esta práctica. Esta relación demuestra entonces que parte de la resistencia de los pobladores en sus territorios está sujeta a la diversificación de las especies que son identificadas y utilizadas por estos como alternativa para el sustento cotidiano de su unidad familiar.

Tabla 2

Relación entre desplazamiento forzado y economías de transición

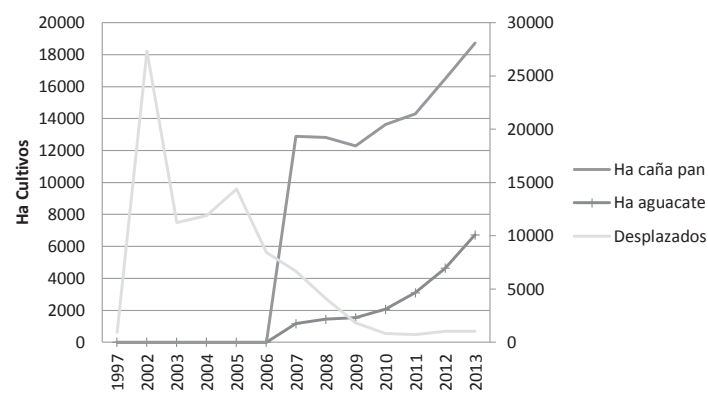

Nota. Adaptada de Desplazamiento en Caldas, por Unidad de Víctimas, 2015, y Evaluaciones agropecuarias municipales Eva, 2007-2013, por Sistema de Estadísticas Agropecuarias, 2015.

Ahora bien, aunque puede haber una relación directa entre el desplazamiento forzado y, en general, el asentamiento del conflicto armado con los bajos niveles de producción de café en este caso, factores como los agropecuarios, ambientales, políticos y técnicos tienen un papel trascendental en la transformación de las necesidades de los pobladores locales, lo que les exige adaptarse a las condiciones para poder quedarse en sus municipios. En ese sentido, a pesar de las dificultades con que los mismos pobladores transitan entre cultivos de tradición y la incursión a nuevas especies, debido a los costos de restablecimiento e implementación de una especie a otra y los conocimientos técnicos que implica algo nuevo, lo realmente importante es establecer una estrategia para que los pobladores no abandonen la agricultura. Dicha estrategia, que convoca fuertes dinámicas de arraigo desde el plano productivo hasta el aspecto sociocultural, es lo que denominamos "agromemoria", en cuyo 
marco aparece la posibilidad de implementar nuevos cultivos como ya hemos mencionado, o por ejemplo, establecer un nivel de asociatividad en el que las instituciones canalicen los recursos e incentiven el trabajo local. De acuerdo con los municipios de estudio, Samaná aporta, por ejemplo, un $42 \%$ de asociaciones dentro de los tres municipios de interés, seguido de Pensilvania y Aguadas con un 29\%, lo que se ha convertido en un lugar común para captar recursos en algunos de los sistemas.

De igual manera, aparece el programa de familias guardabosques (PFGB), iniciativa gubernamental que inicia en Colombia en el 2003, con la intención de

"realizar actividades de conservación, recuperación y preservación de los bosques. Asimismo, apoyar la creación de proyectos productivos sostenibles en las líneas de café, cacao, caucho, palma africana, cultivos forestales, maderables, caña panelera, piscicultura, apicultura, sistemas silvopastoriles y artesanales. Además, promueve la mejora de las relaciones entre vecinos, la formación y/o fortalecimiento de la institución local, el trabajo asociativo" (Giraldo y Lozada, 2008).

Ejemplo de ello es la asociación Agrolinda del municipio de Pensilvania que trabaja sobre el café desde su siembra, cosecha, transformación y comercialización. Los actores locales tienen la posibilidad luego de una transición por diferentes circunstancias de retomar lo que tienen, buscar y aplicar formas de desarrollo que les permitan conservar lo que tradicionalmente ha sido su cultura o simplemente explorar nuevas alternativas que den garantías de sustento, ya que "la tierra nos llama, por eso volvemos" (S. D. Cardona, Líder asociación Agrolinda, comunicación personal, abril, 2015).

Pese a ello, el emergente fenómeno de las asociaciones productivas, que se da principalmente después del conflicto, tiene debilidades estructurales pues en muchos casos no se cuenta con la infraestructura ni con la capacitación respectiva de los actores sociales, que les permita dar un valor agregado real a sus producciones; más aún, cuando el café, la panela, los quesos y otros productos ingresan al país provenientes de otros lugares, a menor precio para el consumidor final y minimizando la economía local porque se aumentan los costos de transacción, por lo tanto, es necesario un direccionamiento objetivo y seguro que les brinde a los actores sociales las garantías necesarias para el buen desarrollo de las cadenas productivas.
Ahora bien, aunque el hecho de crear estrategias que permitan a los pobladores continuar en sus territorios ocurre en su mayoría de manera informal, la adecuación de políticas de tierras y desarrollo resultan determinantes en un país que como Colombia sigue siendo en gran parte rural. En ese sentido, el avance sobre la frontera agrícola incontrolada y, lo que es aún peor, el cambio en los usos del suelo tienen costos sociales, ambientales, productivos y económicos de un peso enorme en la construcción misma de territorio. En lo ambiental, tiene que ver con la pérdida de biodiversidad y de la capa vegetal, lo que puede generar desastres naturales y escasez de recurso hídrico; en lo económico, está relacionado con el costo de oportunidad y con el rendimiento de los sistemas productivos y, desde el punto de vista social, puede haber reducción en la mano de obra, dado el eficientismo de algunos sistemas productivos y el aumento de los costos de producción en desmedro de las necesidades básicas de los pobladores y, aunque es posible que el cambio de uso del suelo incremente la diversificación de especies realizando asociación de ellas o teniendo policultivos como alternativa para romper ciclos fitosanitarios, resistencia y proliferación de algunos agentes que alteran los niveles de producción, además de la reducción de uso de agroquímicos, también es cierto que la transición a nuevos cultivos que en principio les permite quedarse o decidir retornar a sus territorios, a largo plazo genera consecuencias para el desplazamiento de los actores, debido a las implicaciones del conflicto generado por los usos indiscriminados del suelo.

En el departamento de Caldas, por ejemplo, tan solo el conflicto por los usos del suelo corresponde a un total de "305.099,65 hectáreas, lo que indica que el $41 \%$ del área del departamento se está usando con cultivos o actividades inadecuadas o contrarias al tipo de suelo para el que son aptos" (Jiménez, 2013, p. 83).

Adicionalmente, según el Instituto Geográfico Agustín Codazzi (IGAC, 2014):

Caldas tiene el 63 por ciento de su territorio con suelos afectados. Delas 744.560 hectáreas de este departamento cafetero, los conflictos están en 469.072 hectáreas. Caldas es el departamento más sobre utilizado en toda Colombia, con un 55 por ciento de su área afectada por el exceso de agricultura en suelos de alta fragilidad, por pendiente, erosión y procesos de remoción en masa; en este caso la subutilización es baja, solo en un 8 por ciento. El conflicto de suelos se entiende como 
un desequilibrio entre oferta y demanda. La oferta es lo que ofrece el paisaje y sus características para producir mientras que la demanda es lo que hace el hombre con ese suelo. Cuando la demanda es mayor a la oferta se dice que hay conflicto por sobreutilización, pero si la demanda es menor se llama subutilización (p. 2).

La Corporación Nacional de Investigación y Fomento Forestal (ConIf), el Ministerio de Medio Ambiente y la Organización Internacional de Maderas Tropicales (oimt) advierten que en realidad la alta vocación forestal debe ser la actividad con más tránsito en el territorio debido a prácticas de conservación, ya que se posee 460840 ha, es decir, el 58\% del área con esta vocación (Rivera, Vega y Herrera, 1998. pp. 1-3).

\section{Conclusiones}

A pesar de la dificultad de los actores locales por tomar la decisión de quedarse en sus unidades productivas, a causa de los diversos conflictos territoriales, la sustitución de los cultivos tradicionales o las iniciativas de asociatividad se convierten en estrategias que, de alguna manera, les permiten persistir y resistir. Sin embargo, tales prácticas conllevan conflictos del uso del suelo debido a las nuevas alternativas que pueden ofrecer un mayor desarrollo económico.

La agromemoria entendida como los acontecimientos ambientales, económicos, fitosanitarios, políticos y sociales que han marcado la trascendencia del cultivo de café en los escenarios de posconflicto estudiados, muestra la multiplicidad de factores que pueden alterar un sistema productivo, creando la necesidad del cambio y la transformación de la base de una economía, sumado al abandono del arraigo cultural, en muchas ocasiones creado por las expectativas de nuevas dinámicas que van ingresando al departamento.

La interacción que hay entre el cultivo de café y el desplazamiento de actores locales puede llevar a implementar sistemas productivos nuevos que tengan garantías, desarrollo y sostenibilidad que no encuentran en este cultivo al momento de regresar y retomar las actividades agropecuarias. Esto se suma a los factores que han llevado al declive del cultivo; pero que sirven como estrategias de posconflicto, diversificando y fortaleciendo la economía local, además de encaminarse a la asociatividad.

Para ir en pro del uso adecuado del suelo, lo mejor es implementar procesos de reconvención que contengan arreglos agrosilvopastoriles, disminución de carga animal, planes de fertilización adecuados según el tipo de suelo, la disminución y la aplicación de las prácticas culturales más recomendadas para buscar la armonía entre las dinámicas de los pobladores.

\section{Referencias}

Arias, M. e Ibáñez, A. (2012). Conflicto armado en Colombia y producción agrícola: ¿aprenden los pequeños productores a vivir en medio del conflicto? Bogotá: Documentos cede Uniandes. Recuperado de http://ageconsearch. umn.edu/bitstream/146482/2/CEDE\%202012-44.pdf

Bernal, C. (2006). Metodología de la Investigación. México: Pearson Educación. Recuperado de http:// es.slideshare.net/franciscomunoyerrogonzalez/bernal-cesar-a-metodologia-de-la-investigacion-3-ed

Behar. D. E. (2008). Metodología de la investigación. Recuperado de http://museoarqueologico.univalle.edu. co/imagenes/Proyecto\%20de\%20Grado\%201/lecturas/Libro\%20metodologia\%20investigacion.\%20 Libro\%20NB.pdf

Bustillo, A. E. (2006). Una revisión sobre la broca del café, Hypothenemus hampei (Coleoptera: Curculionidae: Scolytinae), en Colombia. Revista Colombiana de Entomología, 32(2), 101-116. Recuperado de http://www. scielo.org.co/pdf/rcen/v32n2/v32n2a01

Caicedo, E. (s. f.). Estructuras de poder político electoral [Monografía]. Misión de Observacion Electoral, MOE y Corporación Nuevo Arco Iris. Recuperado de http:// moe.org.co/home/doc/moe_mre/CD/PDF/caldas.pdf

Federación Nacional de Cafeteros de Colombia, fncc. Recuperado de http://www.federaciondecafeteros.org/ particulares/es/quienes_somos/119_estadisticas_historicas

Giraldo, O. y Lozada, A. (2008). Programa de desarrollo alternativo en Colombia familias guardabosques. Visión desde el enfoque del desarrollo territorial rural. Revista Luna Azul, 27, 60-74. Recuperado de http://www. scielo.org.co/pdf/luaz/n27/n27a04.pdf

Instituto Geográfico Agustín Codazzi, IGAC (2014). IGAC revela "anti ranking" de los departamentos con los mayores conflictos de los suelos en Colombia. Recuperado de http://www.igac.gov.co/wps/wcm/connect/ c8eb398044ab6ec2bbd1ff9d03208435/IGAC+revela. pdf?MOD=AJPERES

Garrido, H. B. (2004). La memoria y la palabra. Acerca del uso de la metodología cualitativa en una investigación sobre mujeres y trabajo en el área de Trancas 
(Tucumán, Argentina). Temas de mujeres, 1(1), 1-23. Recuperado de http://filo.unt.edu.ar/wp-content/ uploads/2015/11/t1_garrido_la_memoria.pdf

Jiménez, R. (Dir.). (2013). Diagnóstico ambiental de Caldas. Plan de Acción 2013-2015. Manizales: Corporación Autónoma Regional de Caldas (Corpocaldas). Recuperado de http://www.corpocaldas.gov.co/ publicaciones/1257/Diagnostico_del_Plan_de_Accion_2013-2015.pdf

Ministerio de Agricultura y Desarrollo Rural [MADR], Gobernación de Caldas, Fondo Nacional de Fomento Hortifrutícola [FNFH], Asociación Hortifrutícola de Colombia [Asohofrucol] y Sociedad de Agricultores y Ganaderos del Valle del Cauca [sAG]. (2006). Plan Frutícola Nacional. Desarrollo de la fruticultura en Caldas. Recuperado de http://www.asohofrucol.com.co/ archivos/biblioteca/biblioteca_101_Pan\%20Frut\%20 CALDAS.pdf

Nates-Cruz, B., García, M., Velásquez, P., Acostas, P., Martínez, C. y Bucheli, M. (2015). Procesos de territorialización de la memoria en escenarios de postconflicto. Caracterización, implicación y lineamientos de políticas en el orden local, regional y nacional. Manizales: Colciencias, Universidad de Caldas, retec, CNM. (Manuscrito).

Rivera, H., Vega, E. y Herrera, G. (Eds.). (1998). Guía para plantaciones forestales comerciales Caldas. (Conif: Serie de documentación No 32). Corporación Nacional de Investigación y Fomento Forestal (Conif), Ministerio de medio ambiente y Organización Internacional de Maderas Tropicales (оIмт) Recuperado de http:// www.itto.int/files/user/pdf/publications/PD39\%2095/ pd\%2039-95-4\%20rev\%201\%20(F)\%20s.pdf

Rivillas, C. Serna, C. Cristancho, M. y Gaitán, A. (2011). La roya del cafeto en Colombia: Impacto manejo y costos del control. Boletín Técnico Cenicafe, 36, pp. 1-53. Recuperado de http://biblioteca.cenicafe.org/bitstream/10778/594/1/036.pdf

Sistema de Estadísticas Agropecuarias. [Agronet]. 2015. Evaluaciones agropecuarias municipales Eva, 20072013. Recuperado de http://www.agronet.gov.co/Paginas/estadisticas.aspx

Unidad de Víctimas. (2015). Desplazamiento en Caldas. Recuperado de http://cifras.unidadvictimas.gov.co/ Home/Desplazamiento 